Bundesgesundheitsbl 2018 $61: 806-811$ https://doi.org/10.1007/s00103-018-2758-3 Online publiziert: 30. Mai 2018

(c) Springer-Verlag GmbH Deutschland, ein Teil von Springer Nature 2018

CrossMark

\author{
Michaela Diercke · Sandra Beermann · Kristin Tolksdorf • Silke Buda • \\ Göran Kirchner \\ Abteilung für Infektionsepidemiologie, Robert Koch-Institut, Berlin, Deutschland
}

\section{Infektionskrankheiten und ihre Codierung}

\section{Was kann sich durch die ICD-11 verbessern?}

\section{Einleitung}

Die Internationale statistische Klassifikation der Krankheiten und verwandter Gesundheitsprobleme (International Classification of Diseases - ICD) wird mittels eines Vorschlagsverfahrens jährlich aktualisiert. Dennoch ist eine regelmäßige umfassende Revision der ICD erforderlich, um diese an den medizinischen Kenntnisstand und den technischen Fortschritt, z.B. die zunehmende digitale Erfassung und Verarbeitung von Gesundheitsdaten und die Weiterentwicklung von IT-Tools für die Codierung, anzupassen. Durch die 11. Revision der ICD, die derzeit in einer Entwurfsversion vorliegt und im Jahr 2019 von der World Health Assembly verabschiedet werden soll, soll zudem die Interoperabilität mit anderen Klassifikationssystemen und Terminologien verbessert werden. Dabei ist die Möglichkeit der Fortschreibung von statistischen Trends, u. a. der Todesursachenstatistik, eine der wichtigsten Prämissen [1].

Ziel dieses Beitrages ist es, am Beispiel der Codierung von Infektionskrankheiten mittels ICD und ihrer Anwendung in der Praxis, insbesondere im Bereich Infektionsepidemiologie, Schwächen zu identifizieren und mögliche Verbesserungen aufzuzeigen, die bei der Entwicklung der ICD-11 und der anschließenden Implementierung in Deutschland berücksichtigt werden sollten.

\section{Codierung von Infektions- krankheiten}

Infektionskrankheiten sind in der 10. Revision der ICD (ICD-10) vor allem im
Kapitel I (Bestimmte infektiöse und parasitäre Krankheiten - A00-B99) zu finden, aber auch weitere Codes, insbesondere in den Kapiteln X (Krankheiten des Atmungssystems - J00-J99, z. B. J09-11 Influenza) und XVI (Bestimmte Zustände, die ihren Ursprung in der Perinatalperiode haben - P00-P96), können zur Verschlüsselung bestimmter Infektionskrankheiten genutzt werden. Die Zuordnung zu den Kapiteln in der aktuellen ICD erfolgt demnach aufgrund unterschiedlicher Konzepte, teilweise nach auslösendem Agens, nach betroffenem Organsystem oder nach Lebensperiode. Schon in den ersten Versionen der ICD wurde deutlich, dass eine Zuordnung zu einzelnen Organsystemen gerade bei den Infektionskrankheiten schwierig ist. In der ersten ICD-Version im Jahr 1893, damals noch das „Internationale Todesursachenverzeichnis" (International List of Causes of Death - ILCD), wurden Infektionskrankheiten wie die Tuberkulose und die Syphilis, bei denen verschiedenste Organmanifestationen möglich sind, als Allgemeinkrankheit und darunter ätiologisch klassifiziert [2].

Die Detailtiefe bei der Codierung der Infektionskrankheiten ist sehr unterschiedlich. Während die z. B. in Deutschland sehr häufige Infektionskrankheit Campylobacter-Enteritis nur als einzelner vierstelliger Code (A04.5) als sonstige bakterielle Darminfektion codiert wird, werden für die Tuberkulose, deren Inzidenz in Deutschland deutlich geringer 
tionen: A41.0 Sepsis durch Staphylococcus aureus in Kombination mit U80.00! Staphylococcus aureus mit Resistenz gegen Oxacillin oder Methicillin). Mit dem Kreuz-Stern-System können neben der Ätiologie (in der Regel der verursachende Erreger) auch Manifestationen verschlüsselt werden (z. B. die G01* Meningitis beianderenortsklassifizierten bakteriellen Krankheiten in Kombination mit A32.1† Meningitis und Meningoenzephalitis durch Listerien). Dies erleichtert z. B. die Identifizierung von invasiven Infektionen (so ist z. B. bei Listeriose nur der Nachweis von Listeria monocytogenes aus Blut, Liquor oder anderen normalerweise sterilen Substraten sowie aus Abstrichen von Neugeborenen gemäß $\$ 7$ Abs. 1 Nr. 29 IfSG meldepflichtig).

\section{Anpassungsbedarf durch neue Erreger}

Im Bereich der Infektionskrankheiten werden immer wieder neue Erreger oder deren Subtypen entdeckt (z.B. das Middle East Respiratory Syndrome Coronavirus - MERS-CoV, neue Influenzaviren) oder bereits bekannte Erreger zeigen eine veränderte Verbreitung (z. B. Zikavirus) oder veränderte Eigenschaften (z.B. Resistenzen). Dafür können bereits vorhandene übergeordnete oder wenig spezifische Kategorien verwendet werden (z.B. B34.2 Infektion durch Coronaviren nicht näher bezeichneter Lokalisation im Fall von MERS). Zudem gibt es spezielle Schlüsselnummern im Kapitel XXII Schlüsselnummern für besondere Zwecke. Dort können z.B. vorläufig bisher nicht belegte Schlüsselnummern für neue Erreger nach Entscheidung durch die WHO genutzt werden, z.B. unter U00-U49 Vorläufige Zuordnungen für Krankheiten mit unklarer Ätiologie und nicht belegte Schlüsselnummern, so wie es für die Zikaviruskrankheit (U06.-) ${ }^{2}$ und das Schwere Akute Respiratorische Syn-

\footnotetext{
2 https://www.aerzteblatt.de/nachrichten/ 65200/ICD-10-Kodierung-fuer-Zika-Virus krankheit (zuletzt abgerufen am 24.04.2018).
}

drom (SARS, U04.-) ${ }^{3}$ umgesetzt wurde. Zusätzlich besteht die Möglichkeit, unter U69.2-! sekundäre Schlüsselnummern für besondere epidemiologische Zwecke (z.B. U69.20! Influenza A/H1N1 Pandemie 2009 (Schweinegrippe), U69.21! Influenza A/H5N1 Epidemie (Vogelgrippe)) zu verwenden. Diese Schlüsselnummern dienen der Spezifizierung besonderer epidemiologischer Ereignisse. Eine Einschränkung des Anwendungszeitraums der Schlüsselnummern kann vom Deutschen Institut für Medizinische Dokumentation und Information (DIMDI) bei Bedarf bekannt gegeben werden. Langfristig kann über das Vorschlagsverfahren ein regulärer ICDCode vergeben werden; dies kann entweder allein in der deutschen Version (ICD-10-GM - German Modification), die für die Codierung im ambulanten und stationären Bereich eingesetzt wird, oder weltweit in der ICD-10-WHO, die in Deutschland für die Verschlüsselung der Todesursachen verwendet wird, umgesetzt werden.

\section{Derzeitige Anwendung der ICD-Klassifikation im Bereich Infektionskrankheiten}

In der Abteilung für Infektionsepidemiologie am RKI werden die mithilfe von ICD codierten Daten zu Infektionskrankheiten in verschiedenen Zusammenhängen verwendet. Die ICD ermöglicht den weltweiten Vergleich von Statistiken zu Infektionskrankheiten, so werden regelmäßig Daten an internationale Organisationen, wie z.B. die WHO, übermittelt. Zunehmend wird die ICD jedoch auch für die Erhebung von Surveillanceund Forschungsdaten eingesetzt, z. B. im Rahmen des Meldewesens (Identifizierung von Meldetatbeständen), aber auch in der syndromischen Surveillance akuter Atemwegsinfektionen, für den Aufbau neuer Surveillance-Systeme sowie für die Evaluation der Datenqualität vorhandener Systeme durch Abgleich mit Sekundärdaten.

\footnotetext{
3 https://www.dimdi.de/static/de/klassi/faq/ icd-10/icd-10-gm/faq_1005.htm_319159481. htm (zuletzt abgerufen am 24.04.2018).
}

Evaluation und Anreicherung der Meldedaten durch Sekundärdaten

Das Meldesystem gemäß Infektionsschutzgesetz (IfSG) ermöglicht neben dem eigentlichen Ziel, der Durchführung von Infektionsschutzmaßnahmen vor Ort durch das Gesundheitsamt, die Fortschreibung von zeitlichen Trends von Infektionskrankheiten. Die Daten zu Infektionskrankheiten, die durch das Meldesystem gemäß IfSG erfasst werden, unterliegen jedoch vielen Limitationen. Insbesondere ist, in Abhängigkeit von der jeweiligen Krankheit, von einer Untererfassung auszugehen [3-5]. Um die Sensitivität des Meldewesens zu evaluieren, ist es daher erforderlich, weitere Datenquellen heranzuziehen. Durch den Abgleich mit Sekundärdaten kann das Ausmaß der Untererfassung quantifiziert werden, dies geschieht in Abhängigkeit von der Datenquelle auch teilweise unter Nutzung von ICD-Codes, z.B. bei der Nutzung von Abrechnungsdaten [4]. Aber z.B. auch Routinedaten der gesetzlichen und privaten Krankenversicherungen, der deutschen Rentenversicherung und der deutschen gesetzlichen Unfallversicherung sowie des morbiditätsorientierten Risikostrukturausgleichs (Morbi-RSA) stellen eine mögliche Datenquelle zur Abschätzung und zum Vergleich von Krankheitshäufigkeiten dar.

Allerdings gehen diese auch mit verschiedenen Limitationen einher. Häufig beziehen sich die Datenquellen nur auf einen Teil der Gesamtbevölkerung; z. B. bilden die Daten von gesetzlichen Krankenkassen nur Behandlungsgeschehen $\mathrm{ab}$, die dem Leistungskatalog der Kassen entsprechen. So werden private Zusatzleistungen und Privatverordnungen für Arzneimittel nicht abgebildet. Manche Datenquellen decken, bedingt durch ihre Mitgliederstruktur, auch nicht die gesamte Bevölkerung Deutschlands ab und sind damit nicht ausreichend repräsentativ. Die Daten werden primär zu anderen Zwecken erhoben, daher sind häufig nicht alle nötigen Angaben verfügbar. Da die ICD-10 in der ambulanten und stationären Versorgung vor allem für Abrechnungszwecke eingesetzt wird, ist z.B. davon auszugehen, dass selbst wenn eine genauere Codierung möglich 
wäre, diese nicht angewendet wird. So kann es sein, dass nur die Hauptdiagnose codiert wird, da diese abrechnungsrelevant ist, weitere wichtige Nebendiagnosen oder ergänzende Codierungen (z.B. Kreuz-Stern-System s. oben) nicht vorgenommen werden, da diese keine Auswirkungen auf die Abrechnung und somit keinen unmittelbaren Nutzen für die Codierenden haben, sondern nur der besseren Darstellung der klinischen Diagnosen bzw. epidemiologischen Bewertung und Auswertung von Daten dienen.

Auch enthalten Routinedaten keine klinischen Angaben, Laborwerte oder Angaben zum Schweregrad der Erkrankung. Eine weitere Limitation kann der fehlende Personenbezug sein, sodass verschiedene Datensätze bezüglich Diagnose und Behandlung nicht zusammengeführt und bestimmte Analysen, wie z.B. Capture-Recapture-Studien, nicht durchgeführt werden können. Bei einigen Datenquellen kann eine Darstellung des zeitlichen Verlaufs ggf. nicht möglich sein, da die Daten nur für einen bestimmten Zeitraum verfügbar sind. Des Weiteren können Änderungen in der Datengenerierung aufgrund von gesundheitspolitischen oder abrechnungstechnischen Regularien eintreten, die beim Jahresvergleich berücksichtigt werden müssen [6]. Vor Durchführung einer Studie ist deshalb immer zu prüfen, ob die avisierten Routinedaten für die Bearbeitung der Studienfrage geeignet sind.

Um das Vorgehen bei der Sekundärdatenanalyse zu standardisieren und transparenter zu machen, wurden entsprechende Berichtsstandards entwickelt, z. B. die STandardisierte BerichtsROutine für Sekundärdatenanalysen (STROSA; [7]). Die Limitationen der Daten sollten jeweils ausführlich diskutiert und bei der Bewertung der Daten berücksichtigt werden.

\section{Todesursachen}

Die heutige ICD hat sich aus dem Internationalen Todesursachenverzeichnis (ILCD, s. oben) entwickelt [2]. Auch heute noch ist die Todesursachenstatistik eine der wichtigsten Anwendungen der

Bundesgesundheitsbl 2018 61:806-811 https://doi.org/10.1007/s00103-018-2758-3

(c) Springer-Verlag GmbH Deutschland, ein Teil von Springer Nature 2018

\section{Diercke · S. Beermann · K. Tolksdorf · S. Buda · G. Kirchner Infektionskrankheiten und ihre Codierung. Was kann sich durch die ICD-11 verbessern?}

\section{Zusammenfassung}

Die Revision der Internationalen statistischen Klassifikation der Krankheiten und verwandter Gesundheitsprobleme (International Classification of Diseases - ICD) geht mit grundlegenden Änderungen der Morbiditätsund Mortalitätsstatistik einher, die auch den Bereich der Infektionskrankheiten betreffen. Die Zuordnung der einzelnen Infektionskrankheiten zu den Kapiteln in der aktuellen ICD-10 erfolgt aufgrund unterschiedlicher Konzepte, teilweise nach auslösendem Agens, nach betroffenem Organsystem oder nach Lebensperiode. Besondere Herausforderungen der Klassifizierung der Infektionskrankheiten bestehen u. a. darin, dass regelmäßig ein Anpassungsbedarf durch neu auftretende Erreger entstehen kann. Außerdem reichen die Angaben hinsichtlich Umfang und Tiefe in der ICD-10 teilweise nicht aus, um epidemiologische Auswertungen der Daten durchzuführen.

Die ICD ermöglicht den weltweiten Vergleich von Statistiken zu Infektionskrankheiten.
Zunehmend wird die ICD jedoch auch für die Erhebung von Surveillance- und Forschungsdaten eingesetzt, z. B. im Rahmen des Meldewesens (Identifizierung von Meldetatbeständen), aber auch in der syndromischen Surveillance akuter Atemwegsinfektionen und für den Aufbau neuer Surveillance-Systeme sowie der Evaluation der Datenqualität durch Abgleich mit Sekundärdaten.

Die Chancen der ICD-11 liegen vor allem darin, dass Infektionskrankheiten eindeutiger codiert werden können und ihre Codierung mehr relevante Informationen für die epidemiologische Bewertung enthält. Durch die hohe Komplexität können jedoch Verzerrungen in den Daten entstehen, die die Fortschreibung der Morbiditäts- und Mortalitätsstatistiken erschweren.

Schlüsselwörter

Infektionskrankheiten · ICD-11 · Codiersysteme $\cdot$ Epidemiologie $\cdot$ Surveillance

\section{Infectious diseases and their ICD coding. What could be improved by the introduction of ICD-11?}

\section{Abstract}

The revision of the International Classification of Diseases (ICD) could change morbidity and mortality statistics significantly, which also affects the area of infectious diseases. Infectious diseases are classified according to their etiology, affected body system or the life period during which the episode occurs. Specific challenges arise from emerging pathogens and the respective necessary adaptation. For epidemiologic analysis ICD10 does not always offer enough additional information.

ICD provides the basis for international comparison of infectious disease morbidity and mortality statistics, but it is also used to collect data for surveillance and research purposes, e.g. the notification system for infectious diseases, syndromic surveillance systems and the evaluation of data quality by using secondary data sources.

ICD-11 offers the chance to better represent epidemiological concepts of infectious diseases by adding more relevant information as affected body system or manifestation. Due to the complexity of coding, ensuring continuity of morbidity and mortality statistics could be challenging.

\section{Keywords}

Infectious diseases - Classification system . ICD · Epidemiology · Surveillance
ICD. Durch die monokausale Statistik, bei der nur das Grundleiden eingeht, wird auch die Erfassung der Todesfälle durch Infektionskrankheiten erschwert [8]. So werden z. B. Sterberaten und Anzahl von Todesfällen durch schwere akute respiratorische Infektionskrankheiten auf der
Grundlage der Todesursachenstatistik unterschätzt [9].

\section{Syndromische Surveillance (z. B. SEED ARE, ICOSARI)}

Für die Erfassung der Influenzaaktivität in der jeweiligen Saison sind neben 
der Datenerfassung über das Meldesystem weitere Erhebungsinstrumente erforderlich. Im Sentinel der Arbeitsgemeinschaft Influenza (AGI) werden seit 2013 akute Atemwegserkrankungen auch über ICD-10-Codes erfasst. Zusätzlich können neben der Einschätzung der Krankheitslast von akuten respiratorischen Erkrankungen auch weitere Parameter zur Einschätzung von Krankheitsschwere, Risikofaktoren, Komplikationen und Folgeerkrankungen (z.B. Lungenentzündung, Beatmungspflicht, akutes schweres Atemnotsyndrom) erfasst und analysiert werden. Diese elektronische Datenerhebung erfolgt im ambulanten Bereich im Rahmen der AGI über das SEED ${ }^{A R E}$-Modul (Sentinel zur elektronischen Erfassung von Diagnosecodes akuter respiratorischer Erkrankungen) sowie in Krankenhäusern über die ICD-10-codebasierte KrankenhausSurveillance schwerer akuter respiratorischer Infektionen - ICOSARI. Im ambulanten Bereich wird die syndromische Surveillance seit Gründung der AGI durch virologische Daten ergänzt [10-12]. Die syndromische Erhebung gewährleistet in Krisensituationen eine stabile Datengrundlage und kann durch die zum Meldesystem ergänzende Datenerhebung die Ärzteschaft und den Öffentlichen Gesundheitsdienst von höheren Meldeaufwänden entlasten [13, 14].

\section{Elektronische Meldung (DEMIS)}

Mit dem Deutschen Elektronischen Melde- und Informationssystem für den Infektionsschutz (DEMIS) soll die elektronische Meldung von gemäß Infektionsschutzgesetz meldepflichtigen Krankheiten und Krankheitserregern implementiert werden. Dabei sollen meldepflichtige Tatbestände unter anderem über die Verwendung der ICD automatisch identifiziert werden und der Meldepflichtige durch das Arzt- oder Krankenhausinformationssystem bereits bei Eingabe der (Verdachts-)Diagnose an seine Meldepflicht erinnert sowie im Meldeprozess unterstützt werden [15]. Der Großteil der Meldungen wird durch Labore erzeugt [16]. Da die Laborinformationssysteme häufig andere Klassifikationssyste- me bzw. Ontologien (z. B. die Logical Observation Identifiers Names and Codes LOINC, Systematized Nomenclature of Medicine - SNOMED, häufig aber auch eigene Nomenklaturen) verwenden, ist die Interoperabilität mit anderen Klassifikationssystemen als ICD ein nicht zu unterschätzender Faktor für die erfolgreiche Implementierung von DEMIS. Dafür ist ein Mapping zwischen den verschiedenen Klassifikationssystemen unter Nutzung semantischer Verfahren erforderlich $[17,18]$.

\section{Anforderungen und Erwartungen an die ICD-11}

Das RKI ist Mitglied in der ICD-11-Arbeitsgruppe des Kuratoriums für Fragen der Klassifikation im Gesundheitswesen (KKG), das derzeit die Implementierung der ICD-11 in Deutschland mit den relevanten Akteuren des Gesundheitssystems vorbereitet. In diesem Rahmen können die im Folgenden genannten Anforderungen sowohl in den internationalen Gremien als auch national eingebracht werden.

Die grundlegende Umstrukturierung der ICD bietet viele Chancen, insbesondere die einer differenzierteren und korrekten Abbildung von Krankheiten. Gleichzeitig birgt die hohe Komplexität Gefahren wegen der durch sie bedingten Verzögerungen bei der Implementierung und der erschwerten Anwendbarkeit in der Praxis [1]. Dabei sollte die ICD-11 im Bereich Infektionskrankheiten folgende Anforderungen erfüllen:

- Möglichkeit zur Fortschreibung der bisherigen Zeitreihen,

- bessere Abbildung der Infektionskrankheiten, insbesondere der Meldetatbestände,

- Flexibilität bezüglich der sich verändernden epidemiologischen Situation und neu auftretender Erreger,

- Ermöglichen einer multikausalen Todesursachenstatistik.

\section{Fortschreibung von Trends}

Die wichtigste Anforderung an die aktualisierte ICD im Bereich Infektionskrankheiten ist die Fortschreibung der bisherigen Zeitreihen für die Gesundheits- berichterstattung, die Veröffentlichung der Meldedaten, die bisher auf ICD10-Basis betriebenen syndromischen Surveillance-Systeme und die Todesursachenstatistik. Gleichzeitig begrenzt diese Anforderung aber die Freiheiten für die grundlegende konzeptionelle Umstrukturierung. Ebenso ist zu beachten, dass sich bereits bei der Einführung einzelner neuer Codes und in noch größerem Maße bei der Implementierung der neuen ICD-Version das Codierverhalten der Anwender ändert und auch bei Abwesenheit inhaltlicher Änderungen Surveillance-Artefakte zu erwarten sind [19]. Andererseits wird durch die ICD-11 nun die Möglichkeit geschaffen, auch seltene Krankheiten zu codieren und dadurch die Gesundheitsberichterstattung für Ereignisse, die bisher nicht ausreichend in den Gesundheitsdaten erfasst werden konnten, zu ermöglichen [20].

\section{Bessere Abbildung der Infektions- krankheiten, insbesondere der Meldetatbestände}

Auch bei der Umstellung von ICD-9 auf ICD-10 hatte man das Ziel verfolgt, das Spektrum bestimmter Krankheiten, darunter das der Infektionskrankheiten, besser zu erfassen. So verbesserte sich zwar die Erfassung deutlich, doch schon bei Einführung der ICD-10 konnten einige Infektionskrankheiten identifiziert werden, die nicht vollständig abgebildet wurden [21]. Gerade komplexe Krankheitsbilder, wie z.B. das der Sepsis, werden durch systematische Fehlcodierungen in den Gesundheitsdaten untererfasst [22]. Zwar können durch eine Validierung die Codes identifiziert werden, die die Krankheit bzw. das Syndrom am besten repräsentieren, allerdings hat auch das Codierverhalten der Anwender Einfluss auf die Validität der Daten [23].

Um die Ressourcen im Gesundheitswesen effektiv einsetzen zu können, sollte in Abhängigkeit von der Krankheit bzw. des auslösenden Erregers eine angemessene Balance zwischen Sensitivität und Spezifität bei der Nutzung der ICD-Codes als Meldeauslöser berücksichtigt werden. Während die Spezifität z.B. für Influenza, Keuchhusten und 
Clostridium-difficile-Infektionen recht gut ist, ist die Sensitivität u. a. aufgrund des variablen und wenig eindeutigen klinischen Bildes teilweise nicht hoch genug [24, 25]. Ein wichtiger Parameter ist hier auch der positive Vorhersagewert („positive predictive value“ - PPV), der angibt, wie viele der eingegangenen Meldungen tatsächliche Fälle werden [26]. Während der PPV der ICD-9 noch sehr stark zwischen den einzelnen Infektionskrankheiten schwankte ([27]: $20,3 \%-96,0 \%)$, wird durch die verschiedenen Dimensionen der ICD-11 gerade in diesem Bereich eine Besserung erwartet. Die Nutzung von semantischen Technologien bei der Entwicklung der ICD-11 gewährleistet, dass die ICD11 leichter um weitere Dimensionen ergänzt, aber auch besser mit anderen Klassifikationssystemen und Ontologien referenziert werden kann, allerdings wird dadurch auch die Komplexität erhöht [28].

\section{Flexibilität}

Gerade im Bereich der Infektionskrankheiten sollte die ICD-11 ausreichend Flexibilität für die sich verändernde epidemiologische Situation, z. B. in Ausbruchssituationen, sowie für neu auftretende Erreger bieten. Hierbei ist zu prüfen, ob dies durch die zeitnahe Umwidmung von nichtvergebenen Schlüsselnummern weltweit oder im Rahmen der Anpassung der German Modification (GM) erfolgen sollte.

\section{Multikausale Todesursachen- statistik}

Mit dem Überarbeitungsprozess der ICD-11 soll auch die multikausale Todesursachenstatistik gestärkt werden. Durch Einführung von unterstützender Software soll das Codierverhalten durch die Bereitstellung von Codierungsalgorithmen möglichst vereinheitlicht und gleichzeitig eine multikausale Todesursachenstatistik ermöglicht werden. Dies kann insbesondere Auswirkungen auf die Analyse seltener Todesursachen haben [29].

\section{Zusammenfassung und Ausblick}

Die Chancen der ICD-11 liegen vor allem darin, dass Infektionskrankheiten zukünftig eindeutiger codiert werden können und ihre Codierung mehr relevante Informationen für die epidemiologische Bewertung enthält. Allerdings sollte bei der Auswertung von ICD-11Codes aus Sekundärdaten berücksichtigt werden, dass die Datenerhebung in der Regel Abrechnungszwecken dient und die Daten hinsichtlich Repräsentativität, Vollständigkeit und Validität Limitationen aufweisen können. Durch die hohe Komplexität sowie Änderungen im Codierverhalten und Codierartefakte können zudem Verzerrungen in den Daten entstehen, die die Fortschreibung der Morbiditäts- und Mortalitätsstatistiken erschweren. Die ICD-11 wird derzeit in Feldstudien getestet und soll auf Grundlage der Ergebnisse angepasst und verbessert werden. Aus Sicht des RKI müssen die Auswirkungen der Änderung der Klassifikation auf die Trends von Infektionskrankheiten, z. B. im Rahmen von Mapping-Studien, evaluiert werden, um eine bessere Interpretation der Zeitreihen zu ermöglichen und ggf. Empfehlungen für die Anpassung der ICD-11 geben zu können.

\section{Korrespondenzadresse}

\section{Diercke}

Abteilung für Infektionsepidemiologie, Robert Koch-Institut

Seestraße 10, 13353 Berlin, Deutschland

dierckem@rki.de

\section{Einhaltung ethischer Richtlinien}

Interessenkonflikt. M. Diercke, S. Beermann, K. Tolksdorf, S. Buda und G. Kirchner geben an, dass kein Interessenkonflikt besteht.

Dieser Beitrag beinhaltet keine von den Autoren durchgeführten Studien an Menschen oder Tieren.

\section{Literatur}

1. Boerma T, Harrison J, Jakob R, Mathers C, Schmider A, Weber S (2016) Revising the ICD: explaining the WHO approach. Lancet 388(10059):2476-2477. https://doi.org/10.1016/S0140-6736(16)31851-7
2. Moriyama IM, Loy RM, Robb-Smith AHT (2011) History of the statistical classification of diseases and causes of death. National Center for Health Statistics, Hyattsville, S12

3. Kuehne $A$, Bouwknegt $M$, Havelaar $A$ et al (2016) HUS active surveillance network Germany. Estimating true incidence of 0157 and non0157 Shiga toxin-producing Escherichia coli illness in Germany based on notification data of haemolytic uraemic syndrome. Epidemiol Infect 144(15):3305-3315

4. Takla A, Wichmann O, Rieck T, Matysiak-Klose D (2014) Measles incidence and reporting trends in Germany, 2007-2011. Bull World Health Organ 92(10):742-749. https://doi.org/10.2471/BLT.13. 135145

5. Schielke A, Takla A, von Kries R, Wichmann O, Hellenbrand W (2018) Marked underreporting of pertussis requiring hospitalization in infants as estimated by capture-recapture methodology, Germany, 2013-2015. Pediatr Infect Dis J 37(2):119-125. https://doi.org/10.1097/INF. 0000000000001698

6. Neubauer S, Kreis K, Klora M, Zeidler J (2017) Access, use, and challenges of claims data analyses in Germany. Eur J Health Econ 18(5):533-536. https://doi.org/10.1007/s10198-016-0849-3

7. SwartE, BitzerEM, Gothe Hetal (2016) A Consensus German Reporting Standard for Secondary Data Analyses, Version 2 (STROSA-STandardisierte BerichtsROutine für SekundärdatenAnalysen). Gesundheitswesen 78(S01):e145-e160

8. Schelhase T, Weber S(2007) Die Todesursachenstatistik in Deutschland. Probleme und Perspektiven. Bundesgesundheitsblatt 50:969. https://doi.org/ 10.1007/s00103-007-0287-6

9. Steppuhn H, Buda S, Wienecke A et al (2017) Zeitliche Trends in der Inzidenz und Sterblichkeit respiratorischer Krankheiten von hoher PublicHealth-Relevanz in Deutschland. J Health Monit 2(3):3-35. https://doi.org/10.17886/RKI-GBE2017-049

10. Buda S, Tolksdorf K, Schuler E, Kuhlen R, Haas W (2017) Establishing an ICD-10 code based SARIsurveillance in Germany - description of the system and first results from five recent influenza seasons. BMC Public Health 17(1):612. https://doi. org/10.1186/s12889-017-4515-1

11. Köpke K, Prahm K, Buda S, Haas W (2016) Evaluation einer ICD-10-basierten elektronischen Surveillance akuter respiratorischer Erkrankungen $\left(S_{E E D}{ }^{A R E}\right)$ in Deutschland. Bundesgesundheitsblatt Gesundheitsforschung Gesundheitsschutz 59(11):1484-1491

12. Zanuzdana A, Köpke K, Haas W (2016) Influenza und ambulant erworbene Pneumonie in hausärztlich tätigen Arztpraxen in Deutschland. Bundesgesundheitsblatt Gesundheitsforschung Gesundheitsschutz59(11):1492-1502

13. Robert Koch-Institut (2017) Nationaler Pandemieplan Teil I - Strukturen und Maßnahmen. RKI, Berlin, S 15-18

14. Robert Koch-Institut (2016) Nationaler Pandemieplan Teil II - Wissenschaftliche Grundlagen. RKI, Berlin, S43-56

15. Diercke M (2017) Deutsches Elektronisches Meldeund Informationssystem für den Infektionsschutz (DEMIS). Epidemiol Bull 30:291-293. https://doi. org/10.17886/EpiBull-2017-03

16. Benzler J, Kirchner G, Diercke M, Gilsdorf A (2014) Das Projekt DEMIS - Konzeptionelle und technische Vorarbeiten für ein elektronisches Meldesystem für den Infektionsschutz. Hygieneinspektor 16(2):20-29 
17. Roldán-García MD, García-Godoy MJ, AldanaMontes JFD (2016) An OWL representation of ICD$10-C M$ for classifying patients' diseases. J Biomed Semantics 7(1):62

18. Streibel O, Kybranz F, Kirchner G (2017) Linked data and ontology reference model for infectious disease reporting systems. In: Panetto Het al (Hrsg) On the Move to Meaningful Internet Systems. OTM 2017 Conferences. Lecture Notes in Computer Science, vol 10574. Springer, Cham

19. Wilson SE, Deeks SL, Rosella LC (2015) Importance of ICD-10 coding directive change for acute gastroenteritis (unspecified) for rotavirus vaccine impact studies: illustration from a populationbased cohort study from Ontario, Canada. BMC Res Notes 8:439. https://doi.org/10.1186/s13104015-1412-5

20. Aymé S, Bellet B, Rath A (2015) Rare diseases in ICD11: making rare diseases visible in health information systems through appropriate coding. Orphanet J Rare Dis 10:35. https://doi.org/10. 1186/s13023-015-0251-8

21. Watzlaf VJ, Garvin JH, Moeini S, Anania-Firouzan P (2007) The effectiveness of ICD-10-CM in capturing public health diseases. Perspect Health Inf Manag 4:6

22. Jolley RJ, Quan H, Jetté N et al (2015) Validation and optimisation of an ICD-10-coded case definitionfor sepsis using administrative health data. BMJOpen 5(12):e9487. https://doi.org/10.1136/bmjopen2015-009487

23. Moore K, Black J, Rowe S, Franklin L (2011) Syndromic surveillance for influenza in two hospital emergency departments. Relationships between ICD-10 codes and notified cases, before and during a pandemic. BMC Public Health 11:338. https://doi.org/10.1186/1471-2458-11-338

24. Jones G, Taright N, Boelle PY et al (2012) Accuracy of ICD-10 codes for surveillance of clostridium difficile infections, France. Emerging Infect Dis 18(6):979-981. https://doi.org/10.3201/eid1806. 111188

25. Moore HC, Lehmann D, de Klerk N et al (2014) How accurate are international classification of diseases-10 diagnosis codes in detecting influenza and pertussis hospitalizations in children? J Pediatric Infect Dis Soc 3(3):255-260. https://doi.org/10 1093/jpids/pit036

26. German RR, Lee LM, Horan JM, Milstein RL, Pertowski CA, Waller MN (2001) Guidelines Working Group Centers for Disease Control and Prevention (CDC). Updated guidelines for evaluating public health surveillance systems: recommendations from the Guidelines Working Group. Mmwr Recomm Rep 50(RR-13):1-35

27. Sickbert-Bennett EE, Weber DJ, Poole C, MacDonald PD, Maillard JM (2010) Utility of international classification of diseases, ninth revision, clinical modification codes for communicable disease surveillance. Am J Epidemiol 172(11):1299-1305. https://doi.org/10.1093/aje/kwq285

28. Tudorache T, Falconer S, Nyulas C, Noy NF, Musen MA (2010) Will Semantic Web Technologies Work for the Development of ICD-11? In:Patel-Schneider PF et al (Hrsg) The Semantic Web - ISWC 2010. ISWC 2010. Lecture Notes in Computer Science, vol 6497. Springer, Berlin, Heidelberg

29. EckertO(2017) VerbesserteQualität dernationalen und internationalen Todesursachenstatistik durch den Kodierkern MUSE. Wirtsch Stat 4:118-130 